\title{
EDITORIAL
}

\section{The Rise of Lung Ultrasound in the Era of COVID-19}

\author{
Shailendra Kumar Motwani ${ }^{1}$, Helen Saunders ${ }^{2 *}$ \\ ${ }^{1} \mathrm{SR}$ in the Cardiothoracic Intensive Care Unit and Anesthesia Department, Mohammed Bin Khalifa \\ Cardiac Center, Riffa, Bahrain. \\ ${ }^{2}$ Consultant in the Cardiothoracic Intensive Care Unit and Anesthesia Department, Mohammed Bin \\ Khalifa Cardiac Center, Riffa, Bahrain.
}

\section{*Corresponding author:}

Helen Saunders, MBCHB, FRCA, Consultant in the Cardiothoracic Intensive Care Unit and Anesthesia Department, Mohammed Bin Khalifa Cardiac Center, Riffa, Bahrain. E-mail: drhelensaunders@gmail.com

Received date: 20 June 2021; Accepted date: 27 June 2021; Published date: December 31, 2021

\begin{abstract}
The current global pandemic caused by the novel coronavirus severe acute respiratory syndrome coronavirus 2 (SARS-COV-2) presents a huge challenge for physicians. Rapid diagnosis, triage and clinical management of these patients is a challenge for physicians but may be aided using lung ultrasound. Lung ultrasound has been in use for over 10 years mainly by critical care practitioners and emergency physicians with variable uptake, but it has gained popularity during the Coronavirus disease-2019 (COVID-19) pandemic as a diagnostic tool and can be easily learned compared to the other ultrasound techniques. Image interpretation is based on identifying artefacts generated by the pleural surface. This technique is non-invasive and can be performed rapidly at the patient's bedside. It has higher accuracy in diagnosis than auscultation and Chest X-ray (CXR) combined. In this article the authors describe the interpretation of lung ultrasound images, particularly in patients with COVID-19 and discuss indications for this technique. Physicians are recommended to gain familiarity with this technique and use of online resources for guidance.
\end{abstract}

Keywords: COVID-19, Lung, SARS-COV-2, Ultrasonography, Triage.

\section{Introduction}

The current global pandemic caused by the novel coronavirus severe acute respiratory syndrome coronavirus 2 (SARS-COV-2) presents a huge challenge for physicians. The virus causes a severe acute respiratory infection in some patients. Rapid diagnosis, triage and clinical management of these patients is a challenge for physicians but may be aided using lung ultrasound.

Lung ultrasound images are generated by the interaction of ultrasound waves with tissue and depend on the difference in properties between solid and air spaces. Soft tissue and air reflect almost all the ultrasound waves meaning structures below the pleura cannot be directly visualised. Most of the interpretation is based on artefacts generated by the pleural surface. This technique is non-invasive and can be performed rapidly at the patients' bedside. It has higher accuracy in diagnosis than auscultation and Chest X-ray (CXR) combined.

Lung ultrasound has been in use for over 10 years mainly by critical care practitioners and emergency 
physicians with variable uptake, but it has gained in popularity during the Corona virus disease-2019 (COVID-19) pandemic as a diagnostic tool and can be easily learned compared to other ultrasound techniques. A Medline search on lung ultrasound and COVID-19 generated over 1000 articles published in the last year on this subject, highlighting the increasing use of this technique with widespread access to point of care ultrasound machines.

The authors believe that the lung ultrasound is a very useful technique especially in the evaluation of COVID-19 patients and to direct their management, it can help reduce their exposure to ionising radiation and it is important for all physicians to gain understanding of the interpretation of these images.

\section{COVID-19 and Respiratory Failure}

COVID-19 is causing a global pandemic with increasing morbidity and mortality- there is a spectrum of illness from mild to severe. The most common symptoms of COVID-19 infection are fever and cough with some patients progressing towards acute respiratory distress syndrome or pneumonia. ${ }^{1}$ Patients with moderate and severe disease will require hospitalisation and intensive therapy including oxygen delivered non-invasively or via ventilation. The most common radiological changes are bilateral patchy infiltrates on the chest $\mathrm{X}$-ray and bilateral ground glass opacities on the Computed tomography (CT) scan.

At the time of writing this paper, there have been a total of 2,46,074 cases of COVID-19 infections in Bahrain with a total of 1,236 fatalities.

\section{Why is Lung Ultrasound Better than the Stethoscope?}

Every doctor is trained in the use of the stethoscope and an examination of the respiratory system is usually formed in this way. However, auscultation is neither sensitive nor specific for respiratory failure. The main sign of respiratory failure is crackles, and they have a sensitivity of $19-67 \%$ and a specificity of $39-96 \%$ and there is also very poor inter observer reliability ( $72 \%$ agreement).

Auscultation does also present some risk to the physician of COVID-19 transmission and if stethoscopes are not adequately cleaned in between patients there may be cross infection however the ultrasound probe can be easily covered with sterile probe covers.

Lung ultrasound can be relatively easily learned with high inter and intra physician reliability and can be used as part of the routine bedside examination of patients daily with today's widespread access to point of care machines. ${ }^{5}$ It has been shown to have an accuracy of $90.5 \%$ in diagnosis of the cause of acute respiratory failure in a study of critically ill patients.

\section{How does Lung Ultrasound Compare to Other Methods such as CT Scanning?}

CT scans are highly sensitive for diagnosis of COVID-19 pneumonia showing typical ground glass opacities, crazy paving and patchy consolidation and have been used as first line investigations; however, this places a huge burden on radiology departments and is not practical for all patients. ${ }^{6}$ Transporting a patient with severe respiratory failure brings risks for the patient and is also time consuming. Lung ultrasound does not involve exposing the patient to ionising radiation.

\section{Interpretation of Lung Ultrasound Images:}

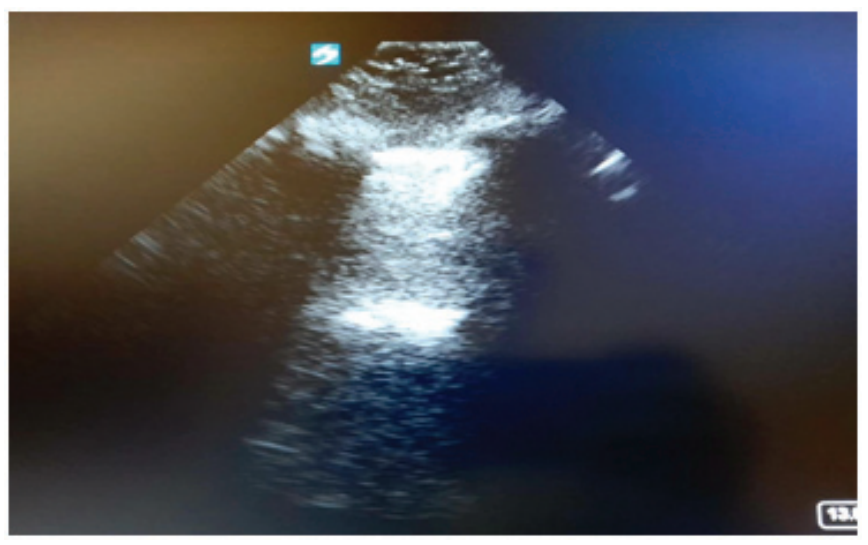

Figure 1: Batwing sign

Chest wall: The first thing to identify is the two rib shadows or the "batwing sign" Figure 1.

Pleural line: In between the rib shadows a bright white line is seen - look for the visceral and parietal pleura sliding back and forth as the patient breathes. This occurs with normal pleura and is absent in pneumothorax, pleural effusion, and dense consolidation, i.e., a non-ventilating lung.

A line: The pleural line reflects most ultrasound 
back to the transducer, this then reflects the waves back and some of the waves bounce back and forth - this can cause additional horizontal lines to be seen equidistant to the pleural lines (Figure 2).

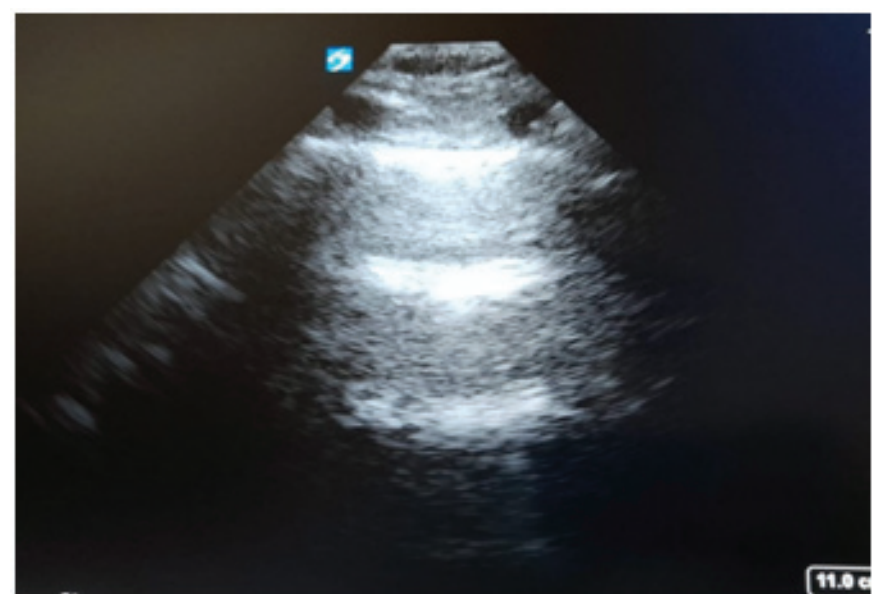

Figure 2: Horizontal Artefact A lines

B lines: These forms when lung tissue thickens or fills with liquid, there are vertical lines which appear ray like. An increased amount of fluid will cause a marked difference in acoustic appearance between this part of the aerated lung and the interlobular septae creating resonance. They start at the pleural surface and travel to at least $18 \mathrm{~cm}$ depth. They appear as a single line but as the fluid increases, they may coalesce leading the lung to look bright. They move with respiration (Figure 3).

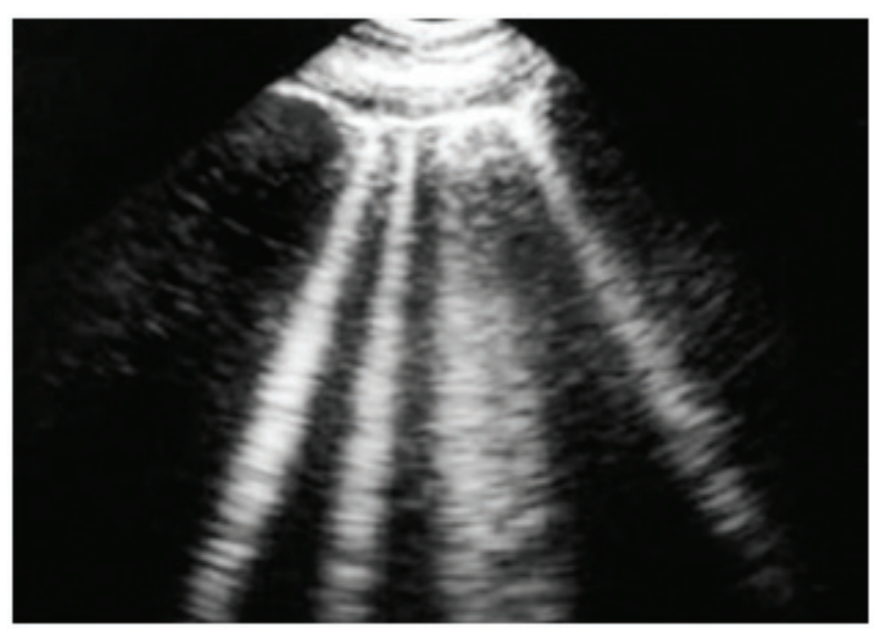

Figure 3: Vertical Artefact B lines

Pleural effusions: Fluid is dark on ultrasound and you may be able to view consolidated lung floating in the effusion (Jellyfish sign) (Figure 4).

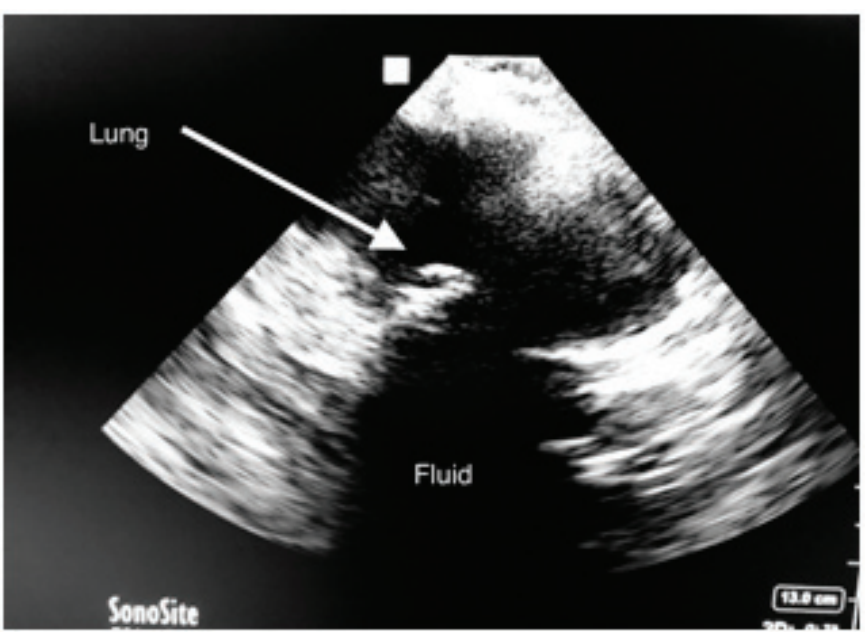

Figure 4: Pleural Effusion with Jellyfish Sign

\section{Lung ultrasound in COVID-19 (Figure 5)}

COVID-19 shows typical ultrasound appearances ${ }^{5}$

- Large number of B lines

- Appearance of shining white lung irregularity of pleural line

- Patchy sub pleural consolidations

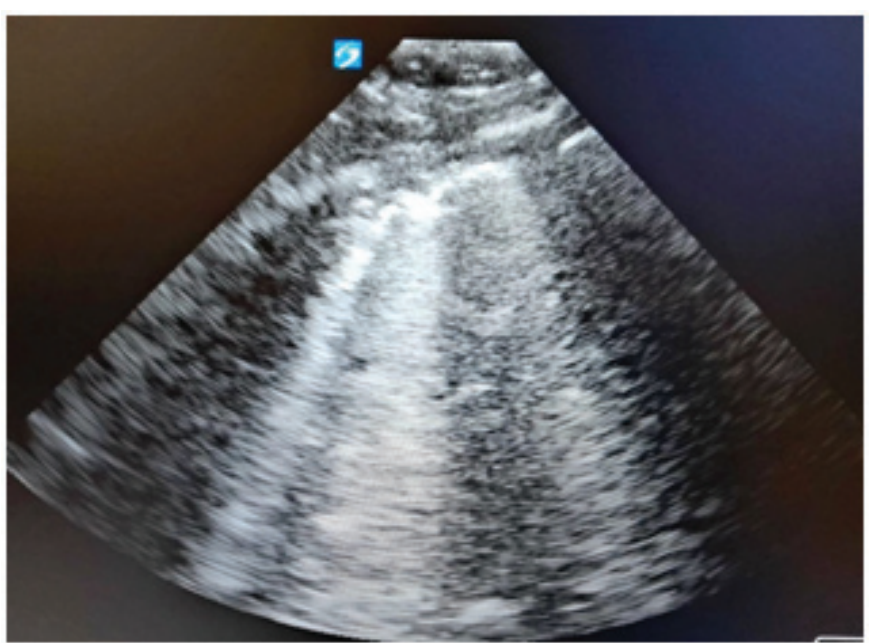

Figure 5: COVID ultrasound showing irregular pleural line and B lines and subpleural consolidation

\section{Which Patients and When Should They Have} Lung Ultrasound?

Lung ultrasound is useful in the emergency room for initial triage to guide the area and degree of care and isolation requirements for the patients. Patients can have a false negative Polymerase chain reaction (PCR) test and in this case lung ultrasound may help to point towards a COVID diagnosis especially 
with an indeterminate CXR. It is very useful in critical care to determine ventilation strategies and other therapeutic procedures such as drain insertion. Also protocols for its use in paediatric and pregnant patients have also been described, minimising these vulnerable patients' radiation exposure. ${ }^{5,6}$

\section{How Can Physicians Get Further Training in Lung Ultrasound?}

Although patients with COVID are not ideal for novices to learn techniques on, many physicians and medical therapists (e.g., physiotherapists) already have basic skills in ultrasound and these can be developed with practise and guidance.

There are many online resources available to help with acquiring knowledge including free access articles and e-learning modules. ${ }^{9,7}$

Medical practitioners have not always stored these images in patient records however this should be encouraged so that records can be kept enabling audit, teaching and for medico legal purposes.

\section{Conclusion}

Although there is no strong evidence so far that lung ultrasound improves outcome its use is increasing as a diagnostic tool.

It is non-invasive, fast, portable, and sensitive. The authors argue it is more sensitive than auscultation and more practical than CT. Familiarity with this technique will allow physicians to gain useful assessment of pathophysiology of the lungs and guide management alongside clinical assessment and use of other diagnostic imaging.

\section{Financial Support}

No grants or sponsorship were received for writing this article.

\section{Conflicts of interest}

Authors have no conflicts of interest to declare.

\section{References}

1. Guan, et al. Clinical Characteristics of Coronavirus Disease 2019 in China. $N$ Engl J Med 2020; 382:1708-1720. https://www.nejm. org/doi/full/10.1056/NEJMoa2002032

2. Miller. Practical Approach to lung ultrasound. BJA education 2016.16(2):39-45https:// academic.oup.com/bjaed/article/16/2/39/28977 63 ? $\log$ in $=$ true

3. Be Aware App Ministry of Health Bahrain 2021. https://healthalert.gov.bh/en/category/beawarebahrain-app

4. Jackson, et al. Lung ultrasound in the COVID-19 pandemic. Postgrad Med J 2020.0:1-6. https:// pmj.bmj.com/content/97/1143/34.long

5. Gargani, et al. When, why and how to use lung ultrasound during the COVID-19 Pandemic: enthusiasm and caution. EHJ 2020;21(9):941948. https://www.ncbi.nlm.nih.gov/pmc/ articles/PMC7314093/

6. Tao, et al. Correlation of chest CT and RT-PCR testing in coronavirus disease 2019 in China. Radiology 2020;296: E32-E40. https://www. ncbi.nlm.nih.gov/pmc/articles/PMC7233399/

7. Mussolino, et al. Lung ultrasound in children with COVID 19; preliminary findings. Ultrasound Med Biol 2020;46(8):2094-2098. https://pubmed.ncbi.nlm.nih.gov/32409232/

8. Moro, et al. How to perform lung ultrasound in pregnant women with suspected COVID-19. UltrasoundObstet Gynecol2020;55(5):593-598. https://pubmed.ncbi.nlm.nih.gov/32207208/

9. Moore S, Gardiner E. Point of care and intensive care lung ultrasound: a reference guide for practitioners during COVID-19. Radiography 2020;26(4): e297-e302. https://pubmed.ncbi. nlm.nih.gov/32327383/

10. NHS E-learning for healthcare: lung ultrasound for COVID-19. https://www.e-lfh.org.uk/ programmes/lung-ultrasound-for-covid-19/ 